\title{
El profesorado francés de Primaria frente a las políticas de rendición de cuentas: una evolución de su profesio- nalismo en un contexto de accountibility subjetiva
} French primary school teachers and accountability policies: how their
professionalism has evolved in a subjective accountability context

Yves Dutercq y Eric Maleyrot'

\section{Resumen}

Nuestra contribución trata de la manera en que el profesorado francés de los centros educativos públicos de educación primaria reaccionan ante las políticas de rendimiento y de responsabilización que se han desarrollado los últimos años. Aspira a comprender en qué medida esto redefine su profesionalismo. En una primera parte, un análisis socio-histórico describe la evolución de las medidas de responsabilización a través de la sucesión de herramientas y de objetivos puestos en práctica para la ayuda individualizada para el éxito del alumnado. Examinamos, a continuación, cómo la responsabilidad del profesionalismo tradicional del profesorado es remodelada por estos nuevos dispositivos, teniendo en cuenta la auto-regulación y la escasa rendición de cuentas de la que es objeto el profesorado. En una segunda parte, nos centramos en la recepción, por parte del profesorado, de dos dispositivos recientes. El primero, es un instrumento externo para medir los resultados del alumnado; el segundo, es un dispositivo que consiste en proponer o poner a su disposición un/a docente complementario en centros educativos que acogen un público desfavorecido. A raíz de las encuestas realizadas sobre estos temas entre 2011 y 2016, nuestro análisis secundario pone a la luz tanto las resistencias como la evolución de las prácticas profesionales que esas políticas de responsabilización suscitan. Para terminar, en una tercera parte, cuestionamos el profesionalismo «interno» del profesorado, frente a la visión del profesionalismo «externo» que espera la institución. Ante una política de responsabilización «suave», los docentes reaccionan o actúan en un contexto que calificamos de accountability subjetiva, dejando translucir dos formas de profesionalidad: una tradicional, que sigue una pedagogía individual, y otra de dimensión colectiva, que acepta rendir cuentas.

\section{Palabras clave}

Accountability, centros educativos de primaria, profesorado francés, prácticas profesionales, profesionalismo docente, responsabilización.

\section{Abstract}

Our paper focuses on how French primary school teachers in state schools are reacting to the performance and accountability policies that have developed in recent years. It seeks to identify to what extent their professionalism is being redefined as a result. In the first section, a socio-historical analysis will describe the developments in accountability measures through the succession of tools and targets put in place as part of an individualized assistance for student achievement. We will examine how the accountability that goes with teachers' traditional professionalism is being reshaped by these new tools, while taking into account self-regulation and the weak accountability that applies to them. In the second section, we will focus on how two recent tools have been received by teachers. The first is an external evaluation device for student results and the second is a tool made available to supplementary teachers in inner-city schools. From studies caried out on these issues between 2011 and 2016, our secondary analysis highlights both the opposition to and changes in professional practices generated by these accountability policies. The third section will call into question the «internal» professionalism of teachers as regards the «external» professionalism expected by the institution. In a so-called «soft» accountability policy, teachers (re)act in a context that we describe as subjective accountability and show two forms of «professionality»: one is traditional and linked to individual pedagogical autonomy and the other has a collective dimension that recognizes accountability.

\section{Keywords}

Accountability, primary school, French teachers, professional practices, teacher professionalism.

\footnotetext{
1 Centre de Recherche en Education de Nantes, Université de Nantes, France. Collaborateur externe du CREN.
} 


\section{Résumé}

Notre contribution porte sur la manière dont les enseignants français de l'école primaire publique réagissent aux politiques de performance et de responsabilisation qui se sont développées ces dernières années. Elle cherche à savoir dans quelle mesure leur professionnalisme s'en trouve redéfini. Dans une première partie, une analyse sociohistorique décrit l'évolution des mesures de responsabilisation à travers la succession des outils et de leurs objectifs mis en place pour l'aide individualisée à la réussite des élèves. Nous examinons alors comment la responsabilité attachée au professionnalisme traditionnel des enseignants est remodelée par ces nouveaux dispositifs, en prenant en compte l'autorégulation et la faible reddition dont ils font l'objet. Dans une seconde partie, nous nous centrons sur la réception par les enseignants de deux dispositifs récents. Le premier est un instrument externe de mesure des résultats des élèves, le second un dispositif de mise à disposition d'un enseignant supplémentaire dans des écoles accueillant un public défavorisé. A partir d'enquêtes réalisées sur ces questions entre 2011 et 2016, notre analyse secondaire met en lumière à la fois les résistances et l'évolution des pratiques professionnelles que ces politiques de responsabilisation suscitent. Enfin, dans une troisième partie, nous questionnons le professionnalisme «interne» des enseignants au regard du professionnalisme «externe» attendu par l'institution. Dans une politique de responsabilisation à régulation «douce», les enseignants (ré)agissent dans un contexte que nous qualifions d'accountability subjective, laissant apparaître deux formes de professionnalité, l'une traditionnelle, attachée à une autonomie pédagogique individuelle, l'autre à dimension collective, acceptant reddition de comptes.

Mołs clés

Accountability, enseignants français, ecole primaire, responsabilisation, professionnalisme enseignant, pratiques professionnelles.

Recibido: 13-03-2017

Aceptado: $11-09-2017$

\section{Introducción}

En los últimos años se han desarrollado considerablemente las políticas que buscan el rendimiento y que suponen la rendición de cuentas en la acción pública en educación, apoyándose en un conjunto de herramientas e instrumentos que hemos tenido la oportunidad de explorar en trabajos recientes (Maroy, 2013; Dutercq et Maroy, 2014, 2017). En Francia, estas políticas nacieron a raíz de la implementación de la Ley orgánica relativa a las leyes de finanza (LOLF) y de la sistematización del pilotaje mediante contratación en el sector de la educación, promoviendo una nueva instrumentalización de la acción pública que se asemeja a una regulación por los resultados (Pons, 2010), sustituyéndose o, más a menudo, superponiéndose a antiguos acuerdos institucionales. Efectivamente, el principio de la rendición de cuentas es antiguo, pero se articula cada vez más con resultados cuantificables y comunicables, gracias al uso de herramientas validadas e institucionalizadas por el Estado o la autoridad central o, de manera más amplia, por el poder regulador: indicadores, evaluación de resultados, contratos, planes de acción, informes de rendimiento, consecuencias individuales o colectivas en términos de apoyo o de control. El desarrollo de estas herramientas tiene un fuerte impacto en el tipo de profesionalismo del personal ya que contribuye a cuestionar su calidad de experto y a poner en tela de juicio su autonomía moral y profesional.

A partir de esta constatación general, nuestra contribución trata de la manera en la que el profesorado francés de los centros educativos públicos de primaria reacciona ante las políticas de rendimiento y de responsabilización.

En una primera parte, proponemos un análisis socio-histórico de la evolución de estas políticas a través de la promulgación de diversas medidas de responsabilización (Dutercq \& Maroy, 2014) que atañen directamente a la enseñanza primaria en Francia. Se centra, muy especialmente, en la ayuda individualizada a favor del rendimiento escolar del alumnado. Describimos la sucesión de herramientas empleadas con este fin por el ministerio francés de la Educación nacional, así como sus objetivos: programa personali- 
zado de ayuda y progreso, programa personalizado de rendimiento escolar, ayuda personalizada, ayuda personalizada complementaria, cursos de puesta al día, acompañamiento educativo. Estas herramientas se articulan a instrumentos de responsabilización más amplios, como las evaluaciones externas nacionales, el proyecto educativo de centro o el cuaderno personalizado de competencias. De esta manera, examinamos cómo la responsabilidad vinculada al profesionalismo tradicional del profesorado de primaria (Geay, 2010; Charles y Cibois, 2010) se ve remodelada con la aparición de nuevos dispositivos, tomando en cuenta la auto-regulación y la escasa rendición de cuentas de las que son objeto (Maroy y Voisin, 2014).

En una segunda parte, nos centraremos en la recepción por parte del profesorado de primaria de dos dispositivos recientes que tratan de modificar y de acrecentar la responsabilidad moral y profesional en lo que concierne el rendimiento escolar del alumnado y en cómo resuelven las dificultades escolares. El primero, es un instrumento externo del ministerio de Educación nacional para medir los resultados del alumnado. El segundo, obedece a una lógica diferente ya que se trata de un dispositivo de atribución de un/a docente complementario, no destinado a una única clase, en centros que acogen un público desfavorecido.

La diversidad de acogida y de uso de estas herramientas nos conduce, en una tercera parte, a interesarnos por las transformaciones de las prácticas profesionales y a cuestionar el profesionalismo reivindicado por el profesorado con respecto al profesionalismo esperado por la institución: ¿qué formas de regulación de la acción del personal de la enseñanza y qué redefinición(es) relativas a su ética (Freidson, 2001; Maroy, 2011) podemos observar? En efecto, si la política de responsabilización se mantiene de forma eminentemente retórica en Francia (Pons, 2014), sin embargo, la sucesión de dispositivos y de medidas conduce al profesorado a reaccionar, o actuar, en un contexto que calificamos de accountability subjetiva y, en particular, a operar transacciones entre las condiciones de eficacia y las condiciones de legitimidad ética de las herramientas desplegadas.

Nuestro artículo se basa en el análisis secundario de los resultados de encuestas que hemos llevado a cabo sobre cada uno de los dos dispositivos de referencia. La metodología empleada en la encuesta, sobre la recepción de la reforma de la evaluación de los resultados del alumnado, ha consistido en el análisis cruzado de textos que emanan del ministerio de la Educación nacional, artículos de prensa especializada y generalista recogidos durante más de tres años, así como de una seria de veinte entrevistas semiestructuradas a responsables locales de la Educación nacional, a directores/as y a docentes de centros educativos de infantil y primaria, los cuales presentan características variadas, en zonas favorecidas y desfavorecidas, en centros públicos y privados (Dutercq \& Lanéelle, 2013). El dispositivo de atribución de un docente complementario ha sido objeto de una encuesta cualitativa longitudinal en dos academias ${ }^{2}$, o distritos educativos, y de una encuesta etnográfica (recopilación de documentos, observaciones, encuestas a docentes y al director/a de centro) en tres escuelas públicas elegidas por su situación de grandes contrastes con respecto a la implementación, el funcionamiento y los efectos del dispositivo (Dutercq \& Maleyrot, 2016, 2017). Esta investigación nos ha permitido poner de relieve las resistencias, las controversias y la evolución de las prácticas engendradas por las políticas francesas de responsabilización moral y profesional en lo que concierne al rendimiento del alumnado.

2 NdT: la academia es la circunscripción administrativa de referencia en la Educación nacional francesa. 


\section{Análisis socio-histórico de la evolución de las políticas de responsabilización}

Desde el final de la década de 1980, inmediatamente después de la promulgación de la importante ley de orientación de 1989, en la educación privada francesa se toman unas medidas orientadas a alcanzar un objetivo central: la adaptación a la diversidad del alumnado como elemento clave para reducir las desigualdades escolares. Por una parte, se trabaja sobre la diversificación pedagógica, y por otra, sobre la evaluación de las competencias del alumnado. En los años siguientes, con el paso del principio de igualdad de tratamiento al de equidad, la noción de diversificación evoluciona con la aparición en los textos oficiales del tema de la individualización.

\subsection{Una sucesión de dispositivos individualizados}

Así es cómo se crea en 1999 un primer dispositivo denominado «programa personalizado de ayuda y progreso». Se trata, entonces, de reforzar la utilidad de la evaluación nacional anual de estudiantes, en vigor desde principios del curso académico 1989-1990, al inicio del tercer año de la educación primaria $(\mathrm{CE} 2)^{3}$, como herramienta para diagnosticar con precisión. Una vez más, se busca implementar una pedagogía diferenciada y una ayuda personalizada para el alumnado que no haya adquirido las competencias básicas en lectura y cálculo. Concretamente, al finalizar la operación nacional de evaluación, y a partir de una reflexión común entre docentes de primaria sobre los resultados del alumnado con dificultades en el dominio de lenguajes, el profesorado construye con cada alumno/a un «programa personalizado de ayuda y progreso», en colaboración con los padres. Cada docente de CE2 se encarga de poner en práctica en su clase este programa individual para el alumnado al que le concierne. Se encarga también de evaluar con regularidad sus aprendizajes y de efectuar los ajustes necesarios. En 2005, la nueva ley de orientación que instaura una «base común de conocimientos y competencias» reemplaza este dispositivo por el «programa personalizado de rendimiento escolan». Este nuevo dispositivo amplía la acción de ayuda a todo el alumnado de la escuela elemental que no parezca poder alcanzar la base común definida para cada ciclo ${ }^{4}$ de aprendizaje. Esta vez, los documentos oficiales indican que es el director o directora del centro educativo quien propone a las familias, o a un responsable legal del alumno/a, que realicen conjuntamente un proyecto personalizado que deberá permitir evaluar regularmente el progreso de ese alumno/a. En la práctica, cada docente encargado de cada estudiante es quien redacta el programa personalizado de rendimiento académico y quien hace partícipes a las familias, y rinde cuentas de las formas de ayuda implementadas durante el tiempo escolar, así como de aquéllas propuestas a la familia, si procede, fuera del horario escolar.

Estas evaluaciones nacionales sistematizadas para todo el alumnado de CE2, y estos dispositivos de ayuda individualizada extendidos a todo el alumnado de la escuela primaria (de los 6 a los 10 años), deben ser considerados como las primeras medidas de responsabilización del profesorado de primaria. A partir de 2007, y de la llegada de Nicolas Sarkozy a la presidencia, los responsables del ministerio del nuevo gobierno pasan de querer modificar las prácticas en el seno de la clase, a través de la "pedagogía diferenciada», a querer obtener estos cambios en el seno de dispositivos específicos, con la esperanza de que tengan un efecto en beneficio de las prácticas ordinarias.

\footnotetext{
3 NdT: tercer año de la escuela elemental en Francia.

4 NdT: la escuela elemental en Francia corresponde a la educación primaria hasta $6^{\circ}$ de EPO, aunque este último curso se imparta en el colegio. Se divide en dos ciclos: 1r ciclo, de 6 a 8 años, y $2^{\circ}$, de 9 a 11
} 
Un primer dispositivo llamado de «ayuda personalizada» se suma al programa personalizado al inicio del año escolar 2008-2009. Éste se inscribe en el proyecto educativo y se extiende al conjunto del alumnado de educación infantil y hasta $3^{\circ}$ de primaria (incluido) que se encuentre en situación de dificultad de aprendizaje. Las dos horas semanales que se le atribuyen, añadidas a las veinticuatro horas de docencia, son impartidas por cada docente en clase, o las coordinadas cuando no es quien se encarga de su alumnado.

Fuera del tiempo escolar obligatorio, tienen lugar otros dos dispositivos que no conciernen al alumnado de la escuela infantil. Los cursos de puesta al día van dirigidos al alumnado de $4^{\circ}$ y $5^{\circ}$ de primaria (9-10 años) en situación de dificultad de aprendizaje, según lo observado por sus docentes. Estos cursos tienen lugar durante las vacaciones de primavera y de verano bajo la forma de módulos de 15 horas y son impartidos por docentes voluntarios. El acompañamiento educativo concierne a los centros de educación prioritaria. Este dispositivo, que constituye una oferta complementaria a la enseñanza obligatoria así como a la ayudad personalizada, se desarrolla al final del día y se trata de una ayuda con los deberes, así como de prácticas deportivas o artísticas. Son docentes voluntarios, asistentes educativos o colaboradores externos. En estos dos últimos dispositivos, el profesorado es remunerado con horas complementarias.

A pesar de las diferentes apelaciones, esta sucesión de dispositivos de individualización no traduce los cambios profundos en las políticas de responsabilización. La instauración de nuevos dispositivos que se suman a los antiguos, o los eternizan, nos muestra mucha más continuidad que ruptura en la acción de los gobiernos sucesivos a pesar de las alternancias políticas. Precisemos también que estos dispositivos se articulan en medidas de responsabilización más amplias: la nuevas evaluaciones nacionales sistematizadas ${ }^{5}$ en segundo curso ( 7 años) y en último curso (10 años) de la escuela elemental a partir del inicio del curso 2008-2009 (analizadas más adelante), así como el cuaderno personal de competencias, implementado para todo el alumnado de 6 a 16 años, en el curso 2010-2011. Este cuaderno tiene una doble función: es una herramienta institucional que demuestra el dominio de siete competencias básicas y es, también, una herramienta pedagógica al servicio de un seguimiento personalizado del alumnado.

\subsection{Una lógica de regulación a través de la responsabilización «suave»}

La acumulación o yuxtaposición de los dispositivos de ayuda en tiempos escolares diferentes ha enturbiado, de alguna forma, la concepción del profesorado sobre el objetivo de las ayudas. ¿Prevención o remedio? ¿Y para qué alumnado? Persiste la duda. En la mayoría de los casos, con la puesta en marcha de la ayuda personalizada, el número de programas escolares personalizados de rendimiento educativo ha disminuido. La ayuda a estudiantes se ha focalizado más en los dispositivos específicos externos a la clase.

Fuera de las horas de clase, su implementación manifiesta interpretaciones más o menos amplias de los documentos oficiales y de las estimaciones relativas y contextuales de las adquisiciones y de las dificultades del alumnado, insiste en esto el informe de 2010-114 (Inspección general de la educación nacional, la Inspección general de la administración de la educación nacional y de la investigación, 2010). Los criterios por los que se considera a un alumnado o a una alumna como con necesidad de recibir este seguimiento se determinan localmente en función de dos elementos: el nivel de sus logros,

Se abandonan las evaluaciones que sirven de diagnóstico al inicio de CE2 (3º de EPO) a partir del año 2006-2007. 
y la convicción del profesorado de poder ayudarle en su progresión. El alumnado se elige según criterios de diversa índole y decisiones más o menos consensuadas en el seno de las escuelas.

De forma general, los inspectores generales constatan pocas modificaciones efectivas en las prácticas docentes, poniendo solo de manifiesto que la diferenciación es más común cuando la clase está constituida de varios niveles, como para los centros educativos pequeños situados en zona rural. Esta diferenciación está rara vez vinculada a la decisión deliberada del profesorado, que trata poco las grandes dificultades, en particular, las del alumnado en situación de gran dificultad en tercer ciclo. El informe subraya, además, que una de las carencias frecuentes viene dada por la escasez de «personalización» de las ayudas:

"En la clase, observamos muy poca enseñanza diferenciada que se sirva de diferentes vías para alcanzar el mismo objetivo para todos. En la ayuda personalizada, la constitución de pequeños grupos con objetivos idénticos es casi la regla y la gestión del grupo es casi siempre la de un pequeño colectivo. Muy raras son las sesiones donde los alumnos se benefician de un momento breve de intercambios realmente personalizados con el maestro, en torno al objetivo trabajado» (Inspección general de la educación nacional, Inspección general de la administración de la educación nacional y de la investigación, 2010: 45).

El director o directora del centro no tiene un estatuto jerárquico en Francia, sino que es a la vez docente, animador pedagógico y coordinador del equipo de docentes. Sin embargo, es quien puede organizar mejor una coherencia global en el centro educativo. Según los inspectores generales, algunos directores han conseguido implementar políticas de centro muy consensuadas, pero la mayoría considera que cada docente es responsable de la ayuda prestada.

La última Ley de orientación sobre la educación de 2013 eterniza estos dispositivos transformando, sin modificar la organización ni cambiar realmente de objetivo, la «ayuda personalizada» en «actividades pedagógicas complementarias. Fundamentalmente, se implementa de forma progresiva el dispositivo «Más maestros que clases», que apunta a reforzar el tutelaje del alumnado intentando evitar la dificultad escolar, y permitir nuevas organizaciones pedagógicas en el seno mismo de la clase (trataremos más adelante esta cuestión).

La evaluación de los efectos de las ayudas sigue siendo una de las mayores debilidades: de manera global, la mejora del «rendimiento educativo» se percibe compleja y delicada en su puesta en funcionamiento ya que abarca tanto el rendimiento escolar como la realización personal o la autoestima. Los responsables territoriales de primaria, cada uno encargado de una circunscripción con varios centros educativos, actúan como incitadores, pero, al disponer de poco tiempo en el terreno, ejercen un control débil y apenas pueden implementar las modalidades de evaluación individuales y sistemáticas que serían necesarias para una buena regulación de los dispositivos.

En definitiva, y a pesar de las reformas sucesivas de la formación de los docentes, su profesionalización no está a la altura de las necesidades generadas por los nuevos dispositivos:

«En el primer nivel, la formación de la pedagogía diferenciada ha sido abundante desde hace un par de décadas (...), a menudo asociada al trabajo sobre la evaluación, en particular la explotación de las evaluaciones nacionales bajo diferentes formas desde 1989. Pero no por ello los maestros se han convertido en expertos. El enriquecimiento de conocimientos - hay una forma de cultura general sobre el tema- no conduce sistemáticamente a aplicaciones prácticas». (Ibid., 49). 
La aparición de nuevos dispositivos forma parte de una lógica de regulación a través de una responsabilización «suave» (Maroy \& Voisin, 2014). Recae, a la vez, en expectativas de reflexividad por parte del profesorado capaz de una auto-regulación de sus prácticas, y en una baja rendición de resultados del alumnado a su superior jerárquico directo, que va más a apoyar que a controlar. Sujetos a las evaluaciones externas nacionales, al proyecto educativo de centro y al cuaderno personal de competencias, estos dispositivos incitan al profesorado, por una parte, a más transparencia y colaboración con respecto a sus colegas y, por otra, a más información y comunicación con las familia del alumnado.

Sin embargo, esta evolución del profesionalismo esperado por el profesorado no parece modificar, o modifica poco, las prácticas pedagógicas en las clases. Los sociólogos dan varias explicaciones que, sumadas, pueden explicar en parte estas dificultades. Para unos, el origen social cada vez menos popular del profesorado, seleccionado con un nivel de Máster (5 años de estudios universitarios) igual que el profesorado de secundaria, suscita un distancia cultural creciente con el alumnado de clases populares, y constituye un factor agravante de las desigualdades sociales (Charles \& Cibois, 2010). Para otros, la relación del profesorado joven con el individualismo, más fuerte que en las antiguas generaciones, le conduce a mantener relaciones más distanciadas con las instituciones: «El relativismo políticoinstitucional, y la preponderancia atribuida a la esfera profesional inmediata, o a la esfera personal, pueden influir también como forma de resistencia a las órdenes institucionales, o como impedimento de la organización colectiva» (Geay, 2010: 89). Es el ejercicio individual o interindividual del oficio el que parece privilegiado a través de la implementación de las cualidades estrictamente relacionales. Se observa «una perpetuación de las matrices corporativas» (Ibid. 83) en los y las maestras jóvenes que adoptan el discurso dominante del grupo profesional, sobre todo a través de temáticas como la articulación enseñanza-educación, la pedagogía diferenciada o el trabajo en grupo. Esta preferencia por lo pedagógico, marcada por una distancia crítica con respecto a las estructuras institucionales, así como la perpetuación de manera débil de los principios políticos durante largo tiempo arraigados en primaria, subrayan las diferencias con el profesorado de secundaria, ya que, en tanto que experto en una disciplina académica, puede reivindicar su autonomía y su profesionalismo (Ibid.). El análisis de dos dispositivos implementados en dos momentos claves de la reforma de la enseñanza primaria es revelador de esta evolución ambivalente de la responsabilización y del profesionalismo del profesorado de primaria.

\section{Recepción por parte del profesorado de Primaria de dos dispositivos de responsa- bilización}

Las dos medidas que estudiaremos aquí pueden parecer a primera vista muy diferentes. Una de ellas hace referencia a la reforma de la evaluación nacional del alumnado de educación primaria promulgada por un gobierno de derechas al empezar el curso 2008-2009, dando como razones la promoción de la eficacia de la escuela y la externalización de su control. La otra medida, un dispositivo de apoyo a las escuelas que acogen a alumnado en dificultad, ha sido implementada por el gobierno de izquierdas al inicio del curso 20132014, con el objetivo de desarrollar el trabajo en equipo y la innovación pedagógica. Sin embargo, las dos convergen, como intentaremos poner de manifiesto, ya que promueven, tanto una como otra, la responsabilización del profesorado y de los centros educativos, bajo modalidades claramente diferentes, sin duda.

\section{1. La reforma de la evaluación del alumnado de educación primaria}

Si la evaluación de la escuela no es una práctica reciente en Francia (Normand, 2005; Pons, 2010), a partir de ahora se inscribe en una lógica de búsqueda de rendimiento y de obligación de resultados, a 
través de la cual el Estado intenta dar a su política de educación una legitimidad que ya no se le atribuye de por sí (Dutercq, 2005; Berrebi-Hoffmann, 2010; Pons, 2011). En particular, el desarrollo de la evaluación en la educación primaria, hasta ahora menor que en la educación secundaria, se justifica con el fin de mejorar la eficacia y la eficiencia de la acción pública. Pero, por las incertidumbres y las tensiones que la acompañan, el profesorado la vive como una prueba, ya que se enfrenta a su concepción del oficio y a ciertos principios que defiende.

Confirmando esta constatación, las nuevas evaluaciones estandarizadas del alumnado suscitan, desde su puesta en funcionamiento, importantes controversias, ya que entran en tensión con las expectativas del personal de los centros educativos de primaria, así como con la de los numerosos usuarios y observadores del sistema educativo, a quienes les parecen inoportunas para este nivel educativo (Duru-Bellat, 2009; Lafontaine, Soussi y Nidegger, 2009). Estas evaluaciones, que tratan de los logros del alumnado en lengua francesa y en matemáticas, en segundo y en el último año de primaria, se desarrollan respectivamente en enero y en mayo, y no a principios de curso, como las anteriores. Sus características provocan así un doble temor entre el profesorado. Por una parte, aunque sean evaluaciones sobre el alumnado, pueden contribuir a evaluarlos al profesorado, ya que tienen lugar durante el curso o al final del año escolar. Por otra parte, una mala evaluación desacredita también al centro y, por consiguiente, al colectivo al que se pertenece. La solidaridad en el seno del equipo lleva pues a tratar de que todos los resultados obtenidos sean satisfactorios y globalmente coherentes para todo el centro educativo.

Estas evaluaciones desestabilizan al profesorado, que las vive con desconfianza: evidentemente, está acostumbrado a ser inspeccionados puntualmente, y a recibir una nota pedagógica, pero una evaluación que trata de la eficacia en su trabajo (medida según los resultados de su alumnado) entra en tensión con sus certezas como profesionales. Quitarle al grupo profesional docente su capacidad de autoevaluarse significa desprofesionalizarlo. También es cuestionar su aptitud a evaluar al alumnado y a sacar una reflexión de los resultados obtenidos. Es, a la vez, romper el contrato tradicional en el que se fundamenta la relación entre el profesorado y sus superiores jerárquicos -hecha de confianza recíproca, de valores y de objetivos comunes- y negar el objetivo más reciente que la institución les ha fijado: hacerse prácticos reflexivos.

Más aún, es poner en tela de juicio otro aspecto de la confianza que la sociedad les ha acordado, a través de la delegación de sus hijos. Esta estima social recae en un sistema de referencia que define el valor de los individuos en función de su contribución a los objetivos que convienen para el mantenimiento de la sociedad. Desde este punto de vista, los y las docentes estiman cumplir convenientemente con sus tareas, a la altura de la definición que su cultura profesional ha construido desde hace tiempo y que no puede tener por jueces más que sus superiores jerárquicos o ellos mismos (Becker, 1952). Por consiguiente, las evaluaciones llevan en sí mismas la sospecha, más insoportable aún en la medida en que se hacen públicas: publicadas y ampliamente comentadas tanto en el interior como en el exterior de la escuela, permitiendo a cualquier persona juzgar el cumplimiento de los objetivos esperados (Duru-Bellat, 2009).

Cuestionar la confianza, o lo que es interpretado como tal por el profesorado -una petición de rendición de cuentas que podría parecer aceptable- obliga a que el profesorado se tenga que justificar y que lo haga en nombre de principios con los que no está forzosamente familiarizado. De hecho, la 
rendición de cuentas se dirige no solo a los superiores jerárquicos de la Educación nacional, como en la tradición de la administración francesa, sino también a los responsables políticos y a la sociedad de la que los medios de comunicación se presentan como portavoces.

Ya no hay ningún obstáculo técnico para la publicación de resultados de las evaluaciones de cada centro, de manera que la competencia entre los centros de primaria está potencialmente abierta, como es el caso de los establecimientos de secundaria, a través de la puesta a disposición del público de los resultados de los exámenes nacionales. Incluso si el ministerio se contenta con publicar los resultados de los establecimientos de secundaria, en particular su valor añadido (Thélot, 1994), y no los compara entre ellos, los medios de comunicación los manejan desde hace tiempo, encargándose de la clasificación, construyendo palmarés y contribuyendo, así, a estimular un funcionamiento fundado en los principios del mercado.

Es más, estas evaluaciones pueden servir para evaluar a docentes y para tener un impacto en su carrera profesional: para algunos, no se trata de ningún riesgo eventual, sino de un cambio de orientación que conduce a hacerlos directamente responsables del rendimiento de sus alumnos/as. Se perfila, de esta manera, el espectro de una accountability dura, del tipo de la que existe en el país, que vincula las pruebas a la remuneración del profesorado y a mantener o cerrar los centros educativos (Carnoy et al., 2003). Ya no se trata de responsabilizar a los individuos dándoles a conocer elementos útiles para mejorar su contribución al rendimiento del alumnado, sino de inculparlos o exculparlos personalmente, como lo explica Martuccelli con respecto a las formas contemporáneas de evaluación: «Con el paso de las organizaciones hacia los individuos, se implementa una lógica inédita de dominación» donde las «desigualdades de resultados se convierten en un asunto de fracaso personali (Martuccelli, 2010: 41-42).

Después de haberlas edulcorado (no se publicaron los resultados), las evaluaciones instauradas en 2008 han sido finalmente abandonadas en 2013, con el cambio de gobierno, para ser sustituidas, al inicio del curso 2015-2016, por una evaluación estandarizada nacional del alumnado con objetivos de diagnóstico, cercana al antiguo modelo. El objetivo es permitir a los equipos pedagógicos identificar las dificultades potenciales de cada estudiante e implementar una respuesta adaptada desde el principio del año escolar. Sin embargo, al contrario que las anteriores evaluaciones nacionales sistematizadas, no se comunican los resultados a los diferentes niveles de la institución, sino que permanecen como herramientas de uso interno, al servicio de los equipos pedagógicos en las escuelas.

\subsection{El dispositivo del docente complementario en las clases de primaria}

El dispositivo «Más docentes que clases» constituye, en sí, una de las principales medidas de la renovación de la educación primaria instaurada por la última ley francesa de orientación de la educación promulgada en 2013 por la izquierda entonces en el poder. La atribución de un/a docente complementario/a en centenares de escuelas repartidas en el territorio nacional, privilegiando las zonas de educación prioritaria, favorece en principio la calidad y el confort de la enseñanza, pero conduce al mismo tiempo a un importante cuestionamiento de las rutinas docentes por medio de una responsabilización supervisada (Spillane, Reiser \& Gomez, 2006). Efectivamente el dispositivo se presenta claramente como una incitación a cuestionar las prácticas pedagógicas, sobre todo por medio de la coordinación y de la organización del trabajo colectivo. Pero constituye también, de manera menos explícita, una herramienta de responsabilización, sobre todo porque su evaluación institucional pide 
no solamente que sea medido el efecto en el rendimiento del alumnado, sino también que se rindan cuentas de su apropiación por parte del equipo docente del centro educativo.

De esta forma, el primer objetivo del dispositivo es poder responder mejor a las dificultades encontradas por el alumnado y ayudarle a efectuar los aprendizajes fundamentales, a la vez que favorece la implementación de nuevas organizaciones pedagógicas, ante todo en el seno de una misma clase, para prevenir las dificultades escolares. El equipo de docentes de los centros educativos de primaria ha sido instituido como garante de la eficacia del dispositivo y responsable de la coherencia de las diversas modalidades de intervención pedagógica (co-intervención en la clase con el o la docente titular ${ }^{6}$, organizarse de manera diferente a la organización habitual por clases, encargarse de grupos de alumnos según sus necesidades). No obstante, son los responsables territoriales los que se encargan de la supervisión y del reajuste regular de la organización o de la acción del profesorado. Si la libertad pedagógica prevalece en la implementación de las intervenciones del docente complementario, la orientación y supervisión de responsables intermediarios reduce los márgenes de maniobra.

Los responsables de la educación primaria tienen grandes expectativas de profesionalización de los equipos: objetivación de las prácticas y cooperación. Pero ¿cuáles son, en la práctica, las consecuencias de la introducción del dispositivo «Más docentes que clases», suponiendo una coordinación de la acción pedagógica, en la manera en la que el profesorado concibe su profesionalidad, definida como un saber respaldado por una deontología (Aballéa, 1997)?

La percepción de la autonomía profesional, así como las interpretaciones de la evaluación del dispositivo, son reveladoras de las formas de profesionalidad del profesorado, emanan de su profesionalismo concebido como ideal y valor de esta profesionalidad. Para una parte de docentes, a pesar de la voluntad afirmada de sus directivos locales en dejarles una gran amplitud de experimentación, el dispositivo constituye una traba a la autonomía pedagógica. Otro grupo de docentes, sin embargo, afirma que dispone de mayor autonomía pedagógica, y muestra una aceptación progresiva de una forma particular de responsabilización, implicando una obligación de rendir cuentas a su superior jerárquico de los efectos objetivados de la implementación del dispositivo.

Esta oposición es el reflejo de la tensión entre el profesionalismo que podemos calificar de interno, definido por los profesionales mismos (docentes, prácticos reflexivos, competentes, que adaptan sus prácticas al alumnado y respetan los procedimientos y las normas establecidas por la profesión), y un profesionalismo llamado externo, en parte definido por agentes externos.

Este nuevo profesionalismo, proveniente de la lógica gerencial de eficacia, se muestra incompatible con el profesionalismo reivindicado por gran parte de docentes, centrado en la instrucción y en la apertura cultural del alumnado, objetivos no sujetos a una evaluación claramente cuantificable. Fruto de ello, emergen dos formas de profesionalidad derivadas del dispositivo «Más docentes que clases»: por un lado, una forma tradicional que considera la obligación de resultados como una subordinación del profesorado, por otro lado, una profesionalidad que puede integrar una herramienta de responsabilización como medio de desarrollo profesional colectivo, contribuyendo sobre todo a mejorar las competencias que se enseñan.

6 Se entiende co-intervención como la intervención de dos docentes en la clase. 


\section{Los efectos de los dispositivos de individualización sobre el profesionalismo docen-} te: ¿̇una accountability subjetiva?

La instalación, en los centros educativos de primaria, de los dispositivos de ayuda pedagógica que privilegian la individualización ha sido acogida de manera muy diversa por el profesorado, en relación con su propia concepción de su misión pero, ante todo, con elementos contextuales que remiten a la situación de su centro, con las relaciones interpersonales del personal que ejerce allí, o con la regulación asegurada por el responsable de circunscripción o la dirección del centro. La organización del trabajo colectivo pedagógico en las escuelas ilustra, desde este punto de vista, la redefinición del profesionalismo de los docentes franceses de primaria.

Nuestros análisis (Dutercq \& Maleyrot, 2017) nos han conducido a observar dos tipos de organización del trabajo colectivo. En algunas escuelas, el director o directora ha creado espacios temporales bien definidos en el calendario del año escolar y consejos de docentes dedicados a llevar a cabo los dispositivos y estar atentos a las dificultades que se puedan encontrar. En otros centros, las reuniones no se anticipan en el año escolar sino que se van programando en función de la actualidad de los proyectos del centro y de las solicitudes institucionales. La multiplicación de los periodos de concertación conduce a veces al director a procurar no saturar al profesorado. Por fin, los equipos docentes se conciertan de manera sistemática, tal día a tal hora, cada semana. En los dos últimos casos, las cuestiones que conciernen los dispositivos de ayuda son abordadas junto a otros puntos en los consejos de docentes o de ciclo.

Desde un punto de vista pedagógico, en el caso del dispositivo «Más docentes que clases», la cointervención ${ }^{7}$ no aparece como una modalidad habitual del profesorado, salvo en las clases de infantil. Si algunos binomios de docentes en los centros de primaria la experimentan, la mayoría prefiere abrir sus clases y trabajar principalmente por grupos creados en función de sus necesidades. Justifican esta opción por reticencias de tipo cultural y político: en efecto están convencidos de que la co-intervención es menos eficaz que la constitución de pequeños grupos. Así, en las clases de doble nivel (donde se agrupa alumnado de edad n y n+1 cuyo programa de adquisiciones escolares es diferente), denuncian la prohibición institucional de desdoblamiento por nivel a favor de una co-intervención que juzgan, bajo varios aspectos, difícil de poner en práctica.

Nos encontramos, pues, con el mismo tipo de comportamiento que hemos observado con la ayuda personalizada en el informe de la Inspección general de 2010 (Inspección general de la educación nacional, la Inspección general de la administración de la educación nacional y de la investigación, 2010). Algunos equipos pedagógicos identifican a ciertos estudiantes como simplemente frágiles, no sin que estén realmente en dificultad, y constituyen grupos a los que les dedican dos horas de ayuda dentro de sus obligaciones profesionales; otros organizan talleres de profundización, incluso de apertura cultural; por fin, otros, escasos, ponen en práctica situaciones complejas que permiten tratar las pequeñas debilidades puntuales del alumnado.

Si se desprende un ideal profesional concebido como profesionalismo interno a la profesión (enseñar es comprometerse a hacer un buen trabajo, de calidad, con el alumnado, para que adquieran los conocimientos y las competencias inscritas en la base común y el programa nacional), se identifican dos declinaciones diferentes de realización y desarrollo profesional, que pueden variar, claro está, de un centro a otro, o de un equipo a otro.

\footnotetext{
Cf. nota 6.
} 
Algunos equipos entienden la co-intervención y la ayuda individualizada como órdenes contrarias a sus convicciones y a sus prácticas habituales de enseñanza. Estos dispositivos institucionales no se perciben como portadores de una plusvalía en su docencia. El trabajo en grupos más pequeños de estudiantes constituye entonces la modalidad privilegiada de las organizaciones pedagógicas, característica de una forma de profesionalidad en la que domina la búsqueda de un confort pedagógico para los docentes aunada a mejores condiciones de aprendizaje para el alumnado.

Al contrario, otros equipos fundamentan su profesionalidad en la búsqueda de una organización pedagógica más variada, a favor de una mejora de sus competencias didácticas y de sus prácticas pedagógicas con el alumnado. Estas modalidades los conducen a privilegiar un trabajo y una regulación de naturaleza colectiva que se aúnan, entonces, con las medidas de responsabilización. La experimentación de la cointervención o, incluso, la organización de situaciones complejas de aprendizaje para tratar las dificultades puntuales del alumnado durante las ayudas personalizadas, remite a la convicción del profesorado de que la innovación pedagógica es más eficaz con el alumnado.

Los y las docentes que se apegan a una forma tradicional de profesionalidad sienten que las peticiones de responsabilización son impuestas por sus superiores jerárquicos (el ministerio, los altos cargos responsables de la educación nacional) o la ideología imperante. Les parece que puedan desposeerlos de su autonomía de decisión (Freidson, 2001) individual o colectiva a la que están muy apegados de manera práctica y simbólica. Para los docentes en busca de una nueva legitimidad profesional, de manera más colectiva, la responsabilización esperada corresponde a una rendición de cuentas aceptable, o puede ser que aceptada, incluso deseable, si no deseada.

Si hablamos de accountability subjetiva, por oposición a una accountability calificada como objetiva, definida e impuesta por la institución a la manera de la gestión centrada en los resultados de Quebec, es porque en Francia la responsabilización queda sujeta ante todo a la percepción del profesorado y a las dos formas de profesionalidad admitidas por las autoridades educativas. Al parecer, contrapuestas, y fundamentadas sobre principios de acción fundamentalmente divergentes, a menudo, en la práctica, son muy dependientes de los contextos de ejercicio más que de convicciones profundamente ancladas. Si la forma tradicional de profesionalidad está muy presente en los centros educativos de primaria donde predomina el trabajo individual, la segunda forma aparece en los centros donde las circunstancias inducen o imponen el trabajo colectivo. Son estos contextos los que relativizan las reacciones a órdenes externas y suscitan, por esta razón, más o menos desconfianza de antemano. Efectivamente, si las evaluaciones estandarizadas por el nivel central constituyen un instrumento de responsabilización mayor que los dispositivos de diversificación y de individualización pedagógicas reguladas de manera «suave», todas estas medidas convergen, sin embargo, en una redefinición del profesionalismo, más o menos impuesta, más o menos aceptada, y más o menos peligrosa, para la autonomía profesional y, más ampliamente, para la condición docente.

\section{Conclusión}

Hemos intentado arrojar luz sobre la manera en que el profesorado francés de la educación primaria pública reaccionan ante las políticas de rendimiento y de responsabilización a través de la implementación de dispositivos institucionales de ayuda individualizada para el rendimiento del alumnado, y la introducción de evaluaciones estandarizadas del alumnado por el ministerio. El análisis socio-histórico de la sucesión de estos dispositivos de individualización en el seno de las clases y en su entorno muestra globalmente pocas modificaciones efectivas en las prácticas docentes. En una lógica de regulación a tra- 
vés de la responsabilización «suave», en la que los responsables territoriales actúan más como incitadores en la implementación de estos dispositivos, el profesorado interpreta los documentos oficiales sin mucho control, y realiza estimaciones de las dificultades de su alumnado en función de su contexto de trabajo.

La focalización en la recepción por el profesorado de estos dos dispositivos, uno de fuerte responsabilización a través de evaluaciones externas, otro de responsabilización más suave a través de la atribución de un instrumento pedagógico, pone de manifiesto las dimensiones éticas y pedagógicas que explican la evolución del profesionalismo docente en primaria.

El dispositivo de evaluación nacional del alumnado es considerado por el profesorado como un ataque directo a su profesionalismo tanto a nivel individual como colectivo. Desconfía de estas evaluaciones procedentes de agentes externos a la profesión, puesto que, aunque midan el rendimiento del alumnado, constituyen también una evaluación de su eficacia en el trabajo y de su responsabilización en el rendimiento de sus estudiantes. El hecho de que esas evaluaciones se hagan públicas lo percibe como una forma de desprofesionalización (Champy, 2012) a la vez por una devaluación de su profesionalidad en tanto práctico/a reflexivo, y por un cuestionamiento del contrato tácito de confianza entre el profesorado, sus superiores jerárquicos y la sociedad.

Por ser pedagógico y cercano al profesionalismo «interno» del profesorado, el dispositivo que atribuye a los centros educativos de primaria un/a docente complementario no provoca las mismas reacciones de hostilidad. Los análisis ponen de manifiesto dos declinaciones del profesionalismo docente: por un lado, bajo la forma tradicional de profesionalidad vinculada a la autonomía pedagógica individual, y para la que la rendición de cuentas sigue siendo una forma de subordinación del profesorado; por otro lado, bajo la forma de una profesionalidad que integre progresivamente cierta rendición de cuentas y que considere la responsabilización como un medio de desarrollo profesional colectivo.

En un contexto en el que las comparaciones internacionales (principalmente PISA) ponen de manifiesto las dificultades recurrentes del sistema educativo francés en reducir las desigualdades sociales y en intentar llevar a todo el alumnado a un buen rendimiento escolar (Consejo nacional de evaluación del sistema escolar, 2016), los altos cargos responsables de la Educación nacional francesa promueven un nuevo profesionalismo «externo» a la profesión docente. Preconizando, como en otros sistemas educativos nacionales, el trabajo colaborativo a favor de un cambio en las prácticas pedagógicas (Fullan, 2007), las medidas de responsabilización implementadas integran el instrumento externalizado de evaluación y los dispositivos específicos para estudiantes con dificultades de aprendizaje. Más que las convicciones arraigadas del profesorado, o la perpetuación de los valores corporativos, nuestros análisis muestran que son los contextos locales los que rinden cuenta de la variabilidad de los efectos de estas medidas en el profesionalismo docente. En una lógica de regulación por medio de la responsabilización «suave», una accountability subjetiva, entendida como una rendición de cuentas «aceptable/ada» y «desable/ada» por los actores individuales o colectivos, puede funcionar. Varía según el contexto del centro educativo, de un centro a otro, interviene a la vez en la percepción del profesorado sobre su autonomía profesional, en particular si es quien toma o no las decisiones sobre sus prácticas pedagógicas según el uso que se haga de las evaluaciones externas, pero también interviene en la acción más o menos proactiva del director del centro y de su responsable directo en la jerarquía administrativa y, sobre todo, del alineamiento o del nivel de integración y de coherencia de la acción de cada uno de los actores (Anderson, 2014). 
Si nos hemos centrado en la respuesta del profesorado francés de educación primaria con respecto a la rendición de cuentas a la institución, el desarrollo de las medidas de responsabilización está dirigido igualmente a la rendición de cuentas a los usuarios. Aplicar los programas de ayuda personalizada o los tiempos específicos de ayuda (ayuda personalizada, actividad pedagógica complementaria, cursos de puesta al día) implica rendir cuentas a las familias no solo de las acciones implementadas a favor de sus hijos/as sino también del progreso realizado por cada estudiante. Articulados a otros instrumentos como el cuaderno personal de competencias y el proyecto educativo de centro, estas herramientas constituyen dispositivos de información que obligan no solo a más transparencia sino también a rendir cuentas a los usuarios/ciudadanos (Ingram \& Schneider, 2006). Cabe constatar que una accountability subjetiva está ya en marcha en relación a las familias. Probablemente se trate de una rendición de cuentas bien aceptada por el profesorado porque tiene lugar en un encuentro singular docentes-familias, muy diferente de una accountability dirigida a la sociedad a través de resultados globales etéreos.

\section{Referencias bibliográficas}

Aballéa, François (1997): "Décentralisation et transformation du travail social”. Sociétés et représentations, 5, $39-49$.

Anderson, S. (2014): “L'individualisme de l'enseignant à l'ère de l'accountability". Education comparée. 11, $187-214$.

Becker, Howard Saul (1952): "Social-Class Variations in the Teacher-Pupil Relationship". Journal of Educational Sociology, 25 (8), 451-465.

Berrebi-Hoffmann, Isabelle (2010): “Evaluation et élitisme: d'une alliance à l'autre”. Cabiers internationaux de sociologie, vol. CXXVIII/CXXIX, 79-90.

Champy, Florent (2012). La sociologie des professions. Paris: Presses Universitaires de France.

Charles, Frédéric \& Cibois, Phiplippe (2010): “L'évolution de l'origine sociale des enseignants du primaire sur la longue durée: retour sur une question controversée”. Sociétés contemporaines, 77, 31-55.

Carnoy, Martin; Elmore, Richard; Siskin, Leslie (2003). The New Accountability: High Schools and High Stakes Tests. New York: Rouledge Farmer.

Conseil national d'évaluation du système scolaire. (2016). Comment l'école amplifie-t-elle les inégalités sociales et migratoires? Dossier de synthèse. Paris: Ministère de l'éducation nationale de l'enseignement supérieur et de la recherche.

Duru-Bellat, Marie (2009). "Évaluation externe du système éducatif et ressenti des acteurs" in Lucie Mottier Lopez \& M. Crahay (dir.). Évaluations en tension. Entre la régulation des apprentissages et le pilotage des systèmes. Bruxelles, Paris: De Boeck.

Dutercq, Yves(2005). Les régulations des politiques d'éducation. Rennes: PUR.

Dutercq, Yves \& Maroy Christian (2014): “Le développement des politiques d'accountability et leur instrumentation dans le domaine de l'éducation : une perspective franco-canadienne". Education comparée, 11.

Dutercq, Yves \& Lanéelle, Xavière (2013): “La dispute autour des évaluations des élèves dans l'enseignement français du premier degré”. Sociologie, 1 (4), 43-62. 
Dutercq, Yves \& Maleyrot, E. (2016). Le dispositif «Plus de maîtres que de classes»: un outil de transformation de l'école? Note du CREN n²4, http://www.cren.univ-nantes.fr/67959790/0/fiche pagelibre/\&RH $=\mathrm{CREN} \& \mathrm{RF}=1333467903410$

Dutercq, Yves \& Maleyrot, Eric (2017). "Le dispositif «Plus de maitres que de classes»: un outil de responsabilisation et de remise en question du professionnalisme enseignant ?” in Yves Dutercq, \& Christian Maroy (dir.). Le travail et le professionnalisme enseignants face aux politiques de responsabilisation. Bruxelles: De Boeck.

Freidson, Eliot (2001). Professionalism. The third logic. Cambridge (UK): Polity Press.

Fullan, Michael (2007). The new meaning of educational change. New-York y Londres: Routledge.

Geay, Bertrand (2010): “Les néo-enseignants face à l'utilitarisme”. Actes de la recherche en sciences sociales, 184, 72-89.

Ingram, H \& Schneider, A.L. (2006). "Policy Analysis for Democracy" in Michael Moran, Martin Rein, Robert E. Goodin (eds.). The Oxford Handbook of Public Policy. Oxford: Oxford University Press.

Inspection générale de l'éducation nationale, Inspection générale de l'administration de l'éducation nationale et de la recherche (2010). Observation et évaluation de l'ensemble des dispositifs d'aide individualisée et d'accompagnement à l'école, au collège et au lycée. Rapport 2010-114. Paris : Ministère de l'éducation nationale.

Maroy, Christian (2011): “Accountability et confiance dans l'institution scolaire”. Education comparée, 5, 127-154.

Maroy, Christian (2013): “L'école à l'épreuve de la performance: Les politiques de régulation par les résultats: trajectoires nationales, usages locaux”. Bruxelles: De Boeck.

Maroy, Christian \& Voisin, Annelise (2014): "Une typologie des politiques d'accountability en éducation: l'incidence de l'instrumentation et des théories de la régulation". Education comparée, 11, 31-57.

Martuccelli, Danilo (2010): “Critique de la philosophie de l'évaluation”. Cahiers internationaux de sociologie, vol. CXXVIII/CXXIX, 27-52.

Normand, Romuald (2005): "La mesure de l'école : de la tradition statistique à la modernité économétrique”. Éducation et Sociétés, 16 (2), 209-226.

Pons, Xavier (2010). Évaluer l'action éducative, Paris: PUF.

Pons, Xavier (2014): “Le «pilotage par les résultats» en éducation en France: archéologie d'une formalisation administrative". Education comparée, 11, 59-81.

Spillane, James P.; Reiser, B. \& Gomez, L. (2006). "Policy implementation and cognition: The role of human, social \& distributed cognition in framing policy implementation” in Meredith I. Honig (ed.). New directions in Educational policy implementation: Confronting complexity. New York: Suny Press.

\section{Notas biográficas}

Yves Dutercq es sociólogo de la educación. Es director del CREN (Centro de Investigación en Educación de Nantes, Francia) y coeditor de Education et sociétés, una revista internacional de sociología de la educación (De Boeck). Su obra trata de las recientes transformaciones en la acción pública educativa. 
Eric Maleyrot es profesor titular de Ciencias de la Educación en el LIRDEF (Laboratorio Interdisciplinario de Investigación en Didáctica, Educación y Formación) de la Universidad Paul Valéry de Montpellier 3. Trabaja sobre la evolución de la función y de la profesionalidad de los actores de la educación y la formación. 